\title{
Stellungnahme und Empfehlung der MWS-Ärztinnen Schweiz zur Organisation des regionalen Notfalldienstes
}

Vorstand der MWS-Ärztinnen Schweiz

Korrespondenzadresse

Dr. med. Gabriele Susanne Merki, Dr. med. Denise Pupato

Sekretariat Medical Women Switzerland MWS

Oberplattenstrasse 73

9620 Lichtensteig
Die Organisation und Aufrechterhaltung des allgemeinen Notfalldienstes ist kantonal, auf Bezirksebene und innerhalb der Notfalldienstkreise geregelt.

Eine Erhebung der Notfalldienstreglemente in den verschiedenen Kantonen und Bezirken ergab sehr unterschiedliche Kriterien für die Verpflichtung zum allgemeinen Notfalldienst. Diese Unterschiede betreffen einerseits den Einschluss der sich am Notfalldienst zu beteiligenden Fachgruppen, andererseits die Kriterien für eine Befreiung vom Notfalldienst. Besonders bei den Kriterien Altersgrenze, Praxis mit Teilzeitpensum, Anzahl Dienste in den ersten Jahren nach Praxiseröffnung sowie Schwangerschaft, Stillzeit und Regelung für Frauen mit Kleinkindern bestehen erhebliche Differenzen. Besonders schwierig für Frauen mit Familien sind die Regelungen in ländlichen Regionen.

Was Schwangerschaft und Stillzeit betrifft, werden elementare, im Arbeitsrecht verankerte Rechte teilweise völlig ausgeklammert.

Heute ist man sich zwar nicht nur in den Städten, sondern auch in den ländlichen Regionen dessen bewusst, dass es für bestimmte frauenspezifische Situationen Regelungen braucht. Der Mangel an Ärzten einerseits und die Zunahme an Frauen in Allgemeinpraxen andererseits erschweren die Akzeptanz von Sonderregelungen für eine spezielle Gruppe und führen teilweise $\mathrm{zu}$ Entsolidarisierung und Spaltung der Kollegen/-innen besonders in kleineren Bezirken.

Eine Umfrage unsererseits hat ergeben, dass viele Notfalldienstreglemente veraltet und in Überarbeitung sind. Weil es nur wenige Frauen in Praxen gab und viele von diesen die Praxistätigkeit mit ihrem Partner teilten, wurden beim Erstellen vieler Reglemente frauenspezifische Punkte nicht berücksichtigt. Es ist davon auszugehen, dass die praktizierenden Frauen zu überlastet sind mit Berufstätigkeit und Familie, um ausreichend auf bestehende Probleme in den Reglementen hinzuweisen.

Die Ärztinnen Schweiz haben deshalb Empfehlungen formuliert für die Überarbeitung der kantonalen Notfallreglemente.

\section{Empfehlungen der MWS-Ärztinnen Schweiz}

Mit einer Teilnahme am normalen Notfalldienst schwierig $\mathrm{zu}$ vereinbarende Situationen umfassen:

Höheres Alter, Schwangerschaft, Mutterschaft, Familie, mangelnde medizinische Qualifikation

Die Altersgrenze für den Belastungsfaktor Dienst sollte für alle Ärzte und Ärztinnen 60 Jahre nicht überschreiten.

Teilzeitarbeit bedeutet selbstverständlich Teilzeitdienst ohne Rundung nach oben. Aufrundungen im Notfalldienst bei Frauen, die sich wegen der Familie für Teilzeitarbeit entschieden haben, sind keine faire Lösung.

Schwangere Frauen, die in der Situation als Praxisinhaberin tagsüber auch arbeiten müssen, sollten obligatorisch ab 32. SSW und, falls erforderlich, auch früher vom Notfalldienst befreit werden.

Während der Stillzeit sollte die Verpflichtung zum Notfalldienst entfallen. Besonders das erste Jahr mit einem Kleinkind bedeutet eine enorme Zusatzbelastung, die nicht delegiert werden kann (viele schlaflose Nächte).

Für die betreuungsintensive Vorschulzeit, d.h. bis zum abgeschlossenen 6. Lebensjahr, ist es wünschenswert, auf Antrag eine Dispensation vom Notfalldienst zu gewähren. Ab Kindesalter 7 Jahre sollten Notfalldienste möglich sein, zumal das Kind alt und reif genug sein sollte, während eines Diensteinsatzes alleine zu Hause zu bleiben.

Alleinstehende Mütter und Väter sind besonders zu schützen, da sie mehr an Zeit investieren müssen, als wenn zwei Elternteile vorhanden sind. Sie sollten keinen Notfalldienst leisten müssen, bis die Kinder zwölfjährig sind.

Viele Bezirke verlangen, dass eine mehrfache Anzahl an Diensten in den ersten 5 Jahren nach Praxiseröffnung geleistet werden muss. Dies kann Frauen in das Dilemma führen, dass die Kombination von Praxistätigkeit und Verwirklichung des Kinderwunsches ad absurdum geführt wird (Studium bis Alter 26 Jahre, Facharztausbildung 5-7 Jahre, Praxiseröffnung mit etwa 
34 Jahren, in den letzten fruchtbaren Jahren enormer Druck durch überhöhte Notfalldienstzahl und Praxisneueröffnung).

Wir empfehlen daher, von solchen Regelungen abzusehen.

Allgemeinärztlicher Notfalldienst sollte im Sinne der Patienten nur von ausreichend ausgebildeten Ärztinnen durchgeführt werden.

\section{Probleme bei der Umsetzung dieser Empfehlungen}

Wer soll all die Notfalldienste übernehmen, die bei der Umsetzung unserer Empfehlungen nicht mehr geleistet werden? Diese Frage ist besonders schwierig zu beantworten für ländliche Bezirke mit geringer Ärztedichte und vielen weiblichen und vielen älteren Ärzten und Ärztinnen. Auch das Familienleben, die Kinder und Ehefrauen männlicher Ärzte sind ein schützenwertes Gut. Trotzdem, falls sich die Regelungen für Familienfrauen und Teilzeittätigkeit in den nächsten Jahren nicht verbessern, werden ländliche Regionen für die Niederlassung von Ärztinnen weniger attraktiv, mit der Folge einer weiteren
Abnahme von niedergelassenen Ärztinnen und einer höheren Belastung der männlichen Kollegen.

\section{Lösungsvorschläge}

Fairerweise sollten die vom Notfalldienst befreiten Ärztinnen eine Ersatzabgabe leisten.

Bezirke mit durch den Notfalldienst sehr belasteten Ärzten stellen zusammen einen Notfallarzt an. Dieser wird mitfinanziert durch die Ersatzabgabe nichtdienstleistender praktizierender Kollegen/-innen.

Auch der einzelne Arzt/die Ärztin erhält die Erlaubnis, für Notfalldienste einen ausreichend ausgebildeten Arzt/ eine ausreichend ausgebildete Ärztin einzustellen (evtl. auch aus dem Ausland).

Reduktion der Dienstbelastung durch Organisation eines Notfalltelefons.

Zusammenarbeit mit dem Zentrumsspital. So könnte beispielsweise ein Assistenzarzt, eine Assistenzärztin des Spitals in Rotation den Dienst für den Bezirk übernehmen. 